\title{
Emotional Well-being
}

National Cancer Institute

\section{Source}

National Cancer Institute. Emotional Well-being. NCI Thesaurus. Code C91778.

The status of one's general temperament and outlook on life. 